
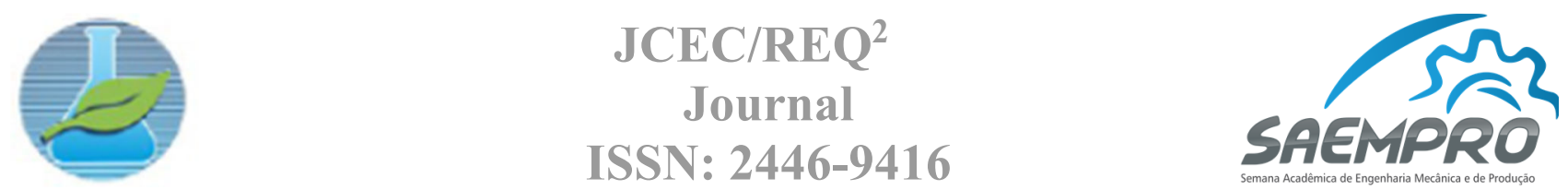

\footnotetext{
"EU, A INDÚSTRIA E O MUNDO"

08 a 11 de novembro de 2016 no campus Viçosa da UFV

Departamento de Engenharia de Produção e Mecânica - DEP

Universidade Federal de Viçosa - UFV
}

\title{
MODELO MATEMÁTICO PARA OTIMIZAÇÃO DA PRODUÇÃO EM UMA EMPRESA PRODUTORA DE PEÇAS AUTOMOBILÍSTICAS
}

\author{
Iago Carrara Roque \\ Universidade Federal de Viçosa, Departamento de Engenharia de Produção e Mecânica \\ $\mathrm{Ph}$. Rolfs s/n - 36570-000 - Viçosa - MG \\ Iago.roque@ufv.br
}

\section{INTRODUÇÃO}

A todo momento é possível se deparar com problemas no qual é demandado grande tempo e esforço para que sejam solucionados manualmente, enquanto é possível utilizar a pesquisa operacional para encontrar soluções ótimas e rápidas. A partir de observações do problema, identificase relações matemáticas que dão origem ao modelo matemático (ARENALES et al., 2007). O objetivo dessa pesquisa é apresentar uma das possibilidades de utilização de um modelo matemático para resolver uma situação que envolve problemas de capacidade e sequenciamento de produção. $\mathrm{Na}$ próxima seção será descrito o problema, na seção 3 será apresentado o modelo matemático, seguido dos resultados obtidos após implementação e, por fim, a conclusão.

\section{DESCRIÇÃO DO PROBLEMA}

O presente estudo de caso ocorreu em uma indústria produtora de peças para carros. Determinado setor da empresa possui duas linhas de produção, sendo que ambas as linhas têm a mesma capacidade e podem produzir os mesmos produtos. Estas linhas de produção fabricam três tipos de peças para automóveis (Produto 1, 2 e 3) em quatro diferentes cores, preto, cinza, azul e verde nas proporções de $40 \%, 20 \%, 10 \%$ e $30 \%$, respectivamente. As linhas de produção possuem diversas estações de trabalho e processos de fabricação, e de acordo com informações de estudos anteriores na empresa, foi constatado que o processo que demandava maior tempo é o de moldagem por injeção, sendo 60 segundos para produção do Produto 1, 40 segundos para o produto 2 e 90 segundos para o Produto 3. Devido a esta etapa demandar o maior tempo da produção e ser considerado o gargalo, o estudo focará apenas neste processo.

Outro fator relevante no processo é a perda de material do processo. De acordo com dados coletados, há um desperdício de pelo menos $2 \%$ de material no processo de moldagem do produto 1 , $3 \%$ do produto 2 e $4 \%$ do produto 3 . Além disso, quando há troca de produto ou cor a ser manufaturado, é realizado o procedimento de set up para realizar modificações na máquina, gerando um tempo ocioso de duas horas na linha em caso de alteração do tipo do produto e 15 minutos para alteração da cor do produto, sem mudança do tipo. Por fim, a empresa funciona em dois turnos de oito horas diárias durante 5 dias na semana.

Com todas as informações obtidas, tem-se o objetivo de encontrar qual a capacidade máxima de ambas as linhas em conjunto durante uma semana, de forma que todos produtos sejam produzidos no período. Após atingir um resultado ótimo, deseja-se organizar a produção de forma que o tempo de set up seja minimizado.

\section{MODELO MATEMÁTICO}


O problema descrito possui dois objetivos. O primeiro é encontrar a maior capacidade de produção, e uma vez encontrado este valor, espera-se obter o resultado que possua menor tempo de set up, por isso a utilização de uma constante próxima de zero para dar peso a cada termo da função. Além disso, algumas variáveis artificiais foram importantes para que algumas restrições fossem modeladas.

Este modelo matemático contém os seguintes parâmetros:

I: Conjunto de 3 produtos; J: Conjunto de 4 cores; K: Conjunto de 2 linhas de produção; $\gamma$ : Constante muito próxima de zero $\left(\gamma=\lim _{n \rightarrow 0} n\right)$; T: Tempo disponível para produzir todos os três produtos.

A seguir são apresentadas as variáveis utilizadas no problema.

$y_{i j k}$ : Número de produtos do tipo $i$ da cor $j$ que entram na linha $k ; y_{i j k} \geq 0$ e inteiro.

$x_{i j k}$ : Número de produtos do tipo $i$ da cor $j$ que saem da linha $k ; x_{i j k} \geq 0$ e inteiro.

$z_{i j k}: 1$ se há produção do tipo $i$ da cor $j$ na linha $k, 0$ caso contrário.

$a_{i k}: 1$ se há produção do tipo $i$ na linha $k, 0$ caso contrário.

$w_{i k}$ : Número de cores do tipo $i$ sendo produzido na linha $k ; w_{i k} \in\{0,1,2,3,4\}$

$l_{k}$ : Tempo de espera na linha $k$.

O problema tem como objetivo maximizar o somatório do número de produtos que saem de ambas as linhas de produção. Uma vez atingindo este objetivo, espera-se minimizar o tempo de espera para troca de produtos e cores na linha de produção.

$$
\max \sum_{i=1}^{3} \sum_{j=1}^{4} \sum_{k=1}^{2} x_{i j k}-\gamma \sum_{k=1}^{2} l_{k}
$$

Para atender os requisitos apresentados na descrição do problema, Seção 2, foram utilizadas as seguintes restrições.

- O número de produtos produzidos de cada tipo deve ser igual.

$\sum_{k=1}^{2} x_{1 j k}=\sum_{k=1}^{2} x_{2 j k}=\sum_{k=1}^{2} x_{3 j k} . \forall j=1,2,3,4$.

- Garantir que a relação entre o número de produtos que entra e o que sai de acordo com as perdas que ocorrem durante a produção.

$x_{1 j k} \leq 0.98 y_{1 j k} . \quad \forall j=1,2,3,4 ; \quad k=1,2$.

$x_{2 j k} \leq 0.97 y_{2 j k} . \quad \forall j=1,2,3,4 ; \quad k=1,2$.

$x_{3 j k} \leq 0.96 y_{3 j k} . \quad \forall j=1,2,3,4 ; \quad k=1,2$.

- Garantir que a quantidade de cada cor será respeitada.

$\sum_{i=1}^{3} \sum_{k=1}^{2} x_{i 1 k}=0.4\left(\sum_{i=1}^{3} \sum_{j=1}^{4} \sum_{k=1}^{2} x_{i j k}\right)$.

$\sum_{i=1}^{3} \sum_{k=1}^{2} x_{i 2 k}=0.2\left(\sum_{i=1}^{3} \sum_{j=1}^{4} \sum_{k=1}^{2} x_{i j k}\right)$.

$\sum_{i=1}^{3} \sum_{k=1}^{2} x_{i 3 k}=0.1\left(\sum_{i=1}^{3} \sum_{j=1}^{4} \sum_{k=1}^{2} x_{i j k}\right)$.

$\sum_{i=1}^{3} \sum_{k=1}^{2} x_{i 4 k}=0.3\left(\sum_{i=1}^{3} \sum_{j=1}^{4} \sum_{k=1}^{2} x_{i j k}\right)$.

- Determina qual o tempo de espera em cada uma das linhas. Os coeficientes utilizados são os tempos de espera em segundos.

$900 \sum_{i=1}^{3}\left(w_{i k}-a_{i k}\right)+7200 \sum_{i=1}^{3}\left(a_{i k}-1\right)=l_{k} \quad \forall k=1,2$.

- Restringe a capacidade de produção de acordo com o tempo disponível. Os coeficientes são os tempos de produção de cada tipo de produto em segundos, e a constante $\mathrm{T}$ é determinada pelo tempo disponível para que todas as peças sejam produzidas. $60 \sum_{j=1}^{4} y_{1 j k}+40 \sum_{j=1}^{4} y_{2 j k}+90 \sum_{j=1}^{4} y_{3 j k}+l_{k} \leq T . \quad \forall k=1,2$.

- Garante que se há produção do tipo $i$ da cor $j$ na linha $k$, a variável $z$ assume valor de 1 , e caso não haja produção, a variável $z$ assume o valor de 0 .

$x_{i j k} \geq z_{i j k} \quad \forall i=1,2,3 ; j=1,2,3,4 ; k=1,2$.

$x_{i j k} \leq \frac{1}{\gamma} \times z_{i j k} \forall i=1,2,3 ; j=1,2,3,4 ; k=1,2$.

- Garante que se há produção do produto $i$ da cor $j$ na linha $k$, a variável $a$ assume o valor de 1, caso não haja produção, a variável $a$ assume o valor de zero.

$\sum_{j=1}^{4} z_{i j k} \geq a_{i k} \quad \forall i=1,2,3 ; k=1,2$. 


$$
\sum_{j=1}^{4} z_{i j k} \leq 4 \times a_{i k} \quad \forall i=1,2,3 ; k=1,2 .
$$

- Determina o quantas cores do tipo $i$ são produzidas na linha $k$.

$$
\sum_{j=1}^{4} z_{i j k}=w_{i k} \quad \forall i=1,2,3 ; k=1,2 \text {. }
$$

\section{RESULTADOS}

O modelo foi implementado no software IBM ILOG CPLEX ${ }^{\circledR}$, e é possível visualizar seu resultado na Tabela 1.1 e na Tabela 2 a quantidade produtos a serem produzidos em cada uma das linhas de produção.

Tabela 1 - Número de produtos de cada tipo produzidos na linha 1.

\begin{tabular}{|l|r|r|}
\hline \multicolumn{3}{|c|}{ Linha 1 } \\
\hline \multirow{2}{*}{ Produto } & \multicolumn{2}{|c|}{ Quantidade } \\
\cline { 2 - 3 } & Entrada & \multicolumn{1}{|c|}{ Saída } \\
\hline Produto 1 (Preto) & 1074 & 1052 \\
\hline Produto 1 (Verde) & 806 & 789 \\
\hline Produto 1 (Azul) & 269 & 263 \\
\hline Produto 1 (Cinza) & 537 & 526 \\
\hline Produto 3 (Preto) & 1096 & 1052 \\
\hline
\end{tabular}

Tabela 2 - Número de produtos de cada tipo produzidos na linha 2.

\begin{tabular}{|l|r|r|}
\hline \multicolumn{2}{|c|}{ Linha 2 } \\
\hline \multirow{2}{*}{ Produto } & \multicolumn{2}{|c|}{ Quantidade } \\
\cline { 2 - 3 } & Entrada & \multicolumn{1}{|c|}{ Saída } \\
\hline Produto 2 (Preto) & 1089 & 1052 \\
\hline Produto 2 (Verde) & 814 & 789 \\
\hline Produto 2 (Azul) & 272 & 263 \\
\hline Produto 2 (Cinza) & 543 & 526 \\
\hline Produto 3 (Azul) & 274 & 263 \\
\hline Produto 3 (Cinza) & 548 & 526 \\
\hline Produto 3 (Verde) & 822 & 789 \\
\hline
\end{tabular}

\section{CONCLUSÃO}

O estudo tinha o objetivo de encontrar o sequenciamento da produção, mas com os resultados obtidos não há necessidade de uma nova modelagem visto que o mesmo pode ser feito de forma manual. Isto é possível pois é um problema simples e que sua maior limitação é o tempo disponível para produção, ou seja, quanto maior o tempo de set up, menor será o tempo disponível para produção. Desta forma, deve ser afirmado tanto para o sequenciamento quanto para a implementação desse modelo, que quando a produção de um produto se inicia, devem ser processadas todas as unidades programadas para ele de acordo com o resultado, até que se inicie a produção de um novo produto. Para estudos posteriores, seria interessante algum modelo que, após sua implementação o resultado determinasse também a sequência de produção, sendo útil para problemas maiores.

\section{AGRADECIMENTOS}

Agradeço ao Professor orientador deste trabalho Gursel A. Suer, pela sua ajuda e disposição guiando este estudo, à estudante Letícia Ferreira de Souza, pela sua contribuição fundamental na realização da pesquisa e construção do modelo matemático, e também aos estudantes Mateus Tartaglia e Gustavo Molino pela assistência prestada na redação deste resumo.

\section{REFERÊNCIAS}

ARENALES et al. "Pesquisa Operacional”. Rio de Janeiro: Campus/Elsevier, 2007. 